\title{
Quantum interference in coherent molecular conductance
}

\author{
Julián Rincón, K. Hallberg \\ Centro Atómico Bariloche and Instituto Balseiro, \\ Comisión Nacional de Energía Atómica and CONICET, 8400 Bariloche, Argentina
}

\author{
S. Ramasesha \\ Solid State and Structural Chemistry Unit, Indian Institute of Science, Bangalore 560 012, India
}

(Dated: November 5, 2018)

\begin{abstract}
Coherent electronic transport through individual molecules is crucially sensitive to quantum interference. Using exact diagonalization techniques, we investigate the zero-bias and zero-temperature conductance through $\pi$-conjugated annulene molecules (modeled by the Pariser-Parr-Pople and Hubbard Hamiltonians) weakly coupled to two leads. We analyze the conductance for different source-drain configurations, finding an important reduction for certain transmission channels and for particular geometries as a consequence of destructive quantum interference between states with definite momenta. When translational symmetry is broken by an external perturbation we find an abrupt increase of the conductance through those channels. Previous studies concentrated on the effect at the Fermi energy, where this effect is very small. By analysing the effect of symmetry breaking on the main transmission channels we find a much larger response thus leading to the possibility of a larger switching of the conductance through single molecules.
\end{abstract}

The possibility of achieving controlled quantum transport through single molecules has become a reality as seen from various successful attempts in different systems [1, 2, 3]. The electronic transport through single $\pi$-conjugated molecules has been studied in several theoretical [4, 5, 6, 7, 8] and experimental [9, 10, 11, 12] works. This important step leads not only to miniaturization of electronic devices but also to the possibility of taking advantage of new physical properties in these systems.

A fundamental physical property in quantum transport is quantum interference, which could be a handle to control conductance through such systems. Constructive and destructive interference play a crucial role which, for most cases, is per se independent of the structure and composition of the molecular bridges to the leads.

Even though changing from constructive to destructive interference might be possible for certain geometries and molecules, disrupting the destructive interference by perturbing the molecule seems to be more dramatic. This has been proposed in two recent theoretical works [6, 7] for the so-called Quantum Interference Effect Transistors (QuIET) based on single annulene molecules, including benzene. In the first paper, the systems are modeled by a Pariser-Parr-Pople (PPP) Hamiltonian 13 using the Ohno parametrization 14 and solved using the self-consistent Hartree-Fock (HF) approximation, while the second work resorts to ab-initio calculations within the LDA and ab-initio approximations. The conductance in both cases is then calculated for the strong coupling limit using the non-equilibrium Green's function 15] and the Landauer-Büttiker formalism [16]. Both studies concentrate on the equilibrium conductance at zero bias and gate voltage $\left(V_{g}=0\right.$, i.e. the off-resonance tunnelling regime) which coincides with the Fermi energy of the leads. However, annulenes have a gap at this energy due mainly to the strong Coulomb interactions present in the molecule $(N=4 n+2$ annulenes like benzene, are closedshell molecules with no level at zero energy even in the absence of interactions). The conductance at zero gate voltage is finite, albeit small, only for strong coupling to the leads [17]. For weak coupling the conductance will be zero and will be appreciable only through the main channels of the molecule which are a few $\mathrm{eV}$ away from the Fermi energy. By analysing the QuIET at the main transmittance channels the switching effect will be much more pronounced and robust as we will show below.

In this work we analyze the linear conductance of a series of annulenes at finite gate voltages and several source-drain lead configurations. For leads set up at 180 degrees (the A configuration in Fig. 1), the system presents completely constructive interference in all transmission channels. However, for other lead configurations, we find different behaviours depending on $N$. For $N=4 n+2$ annulenes we find a reduced conductance through particular channels due to a nearly destructive interferences explained below. When the molecular translational invariance is broken by means of elastic scattering or decoherence factors, we find an abrupt increase in the conductance through these channels due to disruption of destructive interference. This increase is much larger than was found in previous works since the focus is set on a resonance transmission channel through the molecule.

However, for $N=4 n$ annulenes the effect is even more striking: shifting the drain lead by one lattice site from the $\mathrm{A}$ to the $\mathrm{B}$ configuration (see Fig. 1) reduces the transmission to zero. This is, in fact due completely to the destructive interference for $N=4 n$ molecules as explained below. A translational symmetry breaking per- 


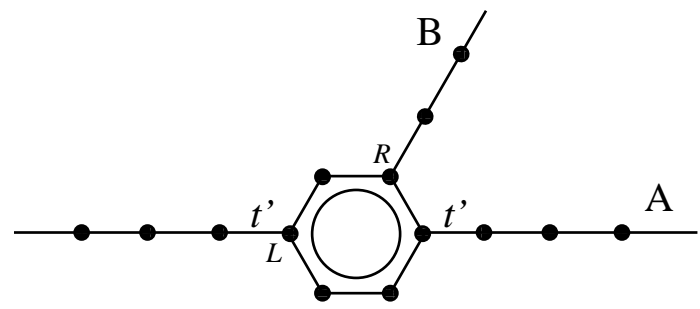

FIG. 1: Schematic representation of the molecules studied numerically in the presence of a gate voltage $V_{g}$, small hybridization to the leads $t^{\prime}$ and two source-drain lead geometries.

turbation will have, as in this case, a huge effect, since the conductance through these channels will increase dramatically from zero to an appreciable value. Hence, such molecules could have important applications. Previous theoretical studies analyze the effect of electron correlation on the structure stability of carbon rings exhibiting competing many-body effects of aromaticity, Jahn-Teller distortion and dimerization [18, 19]. These studies indicate that, under certain conditions, some of the systems considered below could be stabilized. In addition, ring geometries of quantum dots are also potential devices where such a behaviour could be observed.

In this work we consider an annular $\pi$-conjugated molecules with $N$ sites, weakly connected to noninteracting leads in the A or B configurations (Fig. 1). We consider the following Hamiltonian for the system consisting of two leads weakly coupled to the molecule:

$$
H=H_{\text {ring }}+H_{\text {leads }}+H_{\text {links }} .
$$

The first term describes the isolated $\pi$-conjugated molecule, modeled by the PPP Hamiltonian [13], with onsite energy given by a gate voltage $V_{g}$ :

$$
\begin{aligned}
H_{\text {ring }}= & -t \sum_{\langle i j\rangle, \sigma} c_{i \sigma}^{\dagger} c_{j \sigma}+\sum_{i} U_{i}\left(n_{i \downarrow}-\frac{1}{2}\right)\left(n_{i \uparrow}-\frac{1}{2}\right) \\
& -e V_{g} \sum_{i, \sigma} c_{i \sigma}^{\dagger} c_{i \sigma}+\sum_{i>j} V_{i j}\left(n_{i}-1\right)\left(n_{j}-1\right)
\end{aligned}
$$

where the operators $c_{i \sigma}^{\dagger}\left(c_{i \sigma}\right)$ create (annihilate) an electron of spin $\sigma$ in the $\pi$ orbital of the Carbon atom at site $i, n$ are the corresponding number operators and $\langle\cdots\rangle$ stands for bonded pairs of Carbon atoms. The intersite interaction potential $V_{i j}$ is parametrized so as to interpolate between $U$ and $e^{2} / r_{i j}$ in the limit $r_{i j} \longrightarrow \infty$ [14]. In the Ohno interpolation, $V_{i j}$ is given by

$$
V_{i j}=U_{i}\left(1+0.6117 r_{i j}^{2}\right)^{-1 / 2}
$$

where the distance $r_{i j}$ is in $\AA$. The standard Hubbard parameter for $s p^{2}$ Carbon is $U_{i}=11.26 \mathrm{eV}$ and hopping parameter $t$ for $r=1.397 \AA$ is $2.4 \mathrm{eV}[20]$, and all energies are in $\mathrm{eV}$. The second term in Eq.(1) corresponds to two tight-binding semi-infinite chains for the left and right leads. The third term in Eq. (1) describes the coupling of the edge sites of the left and right leads with sites $L$ (left) and $R$ (right) of the system respectively. When the ground state of the isolated ring is non-degenerate, and the coupling $t^{\prime}$ between the leads and the ring is weak $\left(t^{\prime} \ll t\right)$, equilibrium conductance at $0 \mathrm{~K}$ can be expressed to second order in $t^{\prime}$ in terms of the retarded Green's function for the isolated ring between sites $i$ and $j: G_{i, j}^{\mathrm{R}}(\omega)$. For an incident particle with energy $\omega=$ $-2 t \cos k$ and momentum $\pm k$, the transmittance reads 21, 22]:

$$
T\left(\omega, V_{g}\right)=\frac{4 t^{2} \sin ^{2} k|\tilde{t}(\omega)|^{2}}{\left.\left|\left[\omega-\epsilon_{1}+t e^{i k}\right]\left[\omega-\epsilon_{2}+t e^{i k}\right]-\right| \tilde{t}^{2}\right|^{2}},
$$

where $\epsilon_{1(2)}(\omega)=t^{\prime 2} G_{L L(R R)}^{\mathrm{R}}(\omega), \tilde{t}(\omega)=t^{\prime 2} G_{L R}^{\mathrm{R}}(\omega)$, play the role of a correction to the on-site energy at the extremes of the leads and an effective hopping between them respectively. The conductance is $G=$ $\left(2 e^{2} / h\right) T\left(\mu, V_{g}\right)$, where $\mu$ is the Fermi level, which we set to zero (half-filled leads) and $V_{g}$ enters implicitly through the Green's functions.

In our study, the interacting system with $N$ sites is solved exactly using numerical techniques (Lanczos or Davidson) to diagonalize huge matrices, and the Green's functions are obtained straightforwardly. The correlations are, thus, treated in an exact manner.

We now analyze the two possible scenarios for annulenes with $N=4 n+2$ and $N=4 n$ with two terminals. For the first case, the allowed total momentum quantum numbers are $k=2 r \pi /(4 n+2)=r \pi /(2 n+1)$, with $r$ an integer, while for the second case the allowed momenta are $k=2 r \pi / 4 n=r \pi / 2 n$. For a two-terminal set up, wave functions travelling through both branches of the molecule will interfere producing different interference patterns depending on the positions of the leads. The phase difference will be momentum times the difference in the lenghts of the two trajectories (in units of the C-C separation): $\Delta \phi=k \Delta x$.

Specializing to benzene $(N=6)$, for leads in the "para" position, $\Delta x=0$ and the waves are in phase, interfering constructively (Fig. 2). However, in the "meta" position $(\Delta x=2)$, the interference will depend on the $k$ value of the particular channel. For the highest occupied molecular orbital (HOMO) and lowest unoccupied molecular orbital (LUMO) the phase differences will be $\Delta \phi=2 \pi / 3$ and $\Delta \phi=4 \pi / 3$ respectively and the interference will reduce the amplitude in these channels. For benzene configuration of the leads producing a completely destructive scenario for these channels does not exist. Previous experimental and theoretical works [23, 24] noted that the transmission through benzene in the "meta" configuration is much lower than that through the "para" position.

There is no transmission channel at $V_{g}=0$ since the density of states of the bare molecule at that energy is 


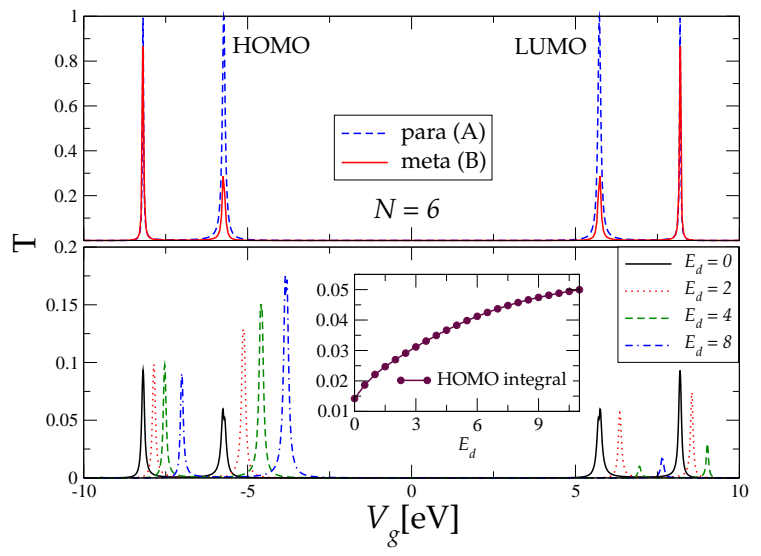

FIG. 2: Top: Transmittance through a benzene molecule for the "para" (A substitution in Fig. 1) (broken line) and "meta" (B substitution in Fig. 1) (full line) configurations as a function of the gate voltage measured from the Fermi energy. Here $t^{\prime}=0.4$. Bottom: Same for the "meta" configuration in the presence of different on-site potentials which break the translational invariance. Here a finite Lorentzian width $\eta=0.03$ and $t^{\prime}=0.1$ have been taken for visualization purposes. Inset: evolution of the area under the transmission peak through the HOMO as a function of local energy.

zero. A detailed analysis of quantum interference effects in benzene was carried out for the large coupling regime [25] where interesting effects appear at zero energy and finite bias [26, 27] at weak coupling.

What would happen if the translational symmetry is broken by an external perturbation? This question was first addressed in [6] by introducing a local energy $(\Sigma)$, at one site in benzene, the real part of $\Sigma$ would produce elastic scattering and its imaginary part, decoherence. In that study, the focus was at the Fermi energy of the leads (set to zero) where the observed effect is small. However, by studying the effect of external perturbations on the main transmittance channels such as the HOMO and LUMO, we find a much larger response. We show these results in the bottom part of figure 2, where an additional diagonal energy is added to the site to the right of the B ("meta") position (the effect is not qualitatively dependent on this position). It is clearly seen that, in this case, the small peak corresponding to the HOMO level develops and grows as the local energy is increased, disrupting the translational symmetry responsible for the destructive interference (see inset).

For larger $4 n+2$ rings, the momentum of the HOMO level approaches $\pi / 2$ and the interference becomes more destructive. This is shown for a 10 site annulene in figure 3 , where the peaks for the $\mathrm{B}$ position of the leads are much smaller than those corresponding to benzene (Fig. 2 , top). Growth of the HOMO peak with a local potential energy is larger in this case than in benzene.

However, for annulenes with $4 \mathrm{n}$ atoms, depending on the total momentum of the particular channel, the inter-

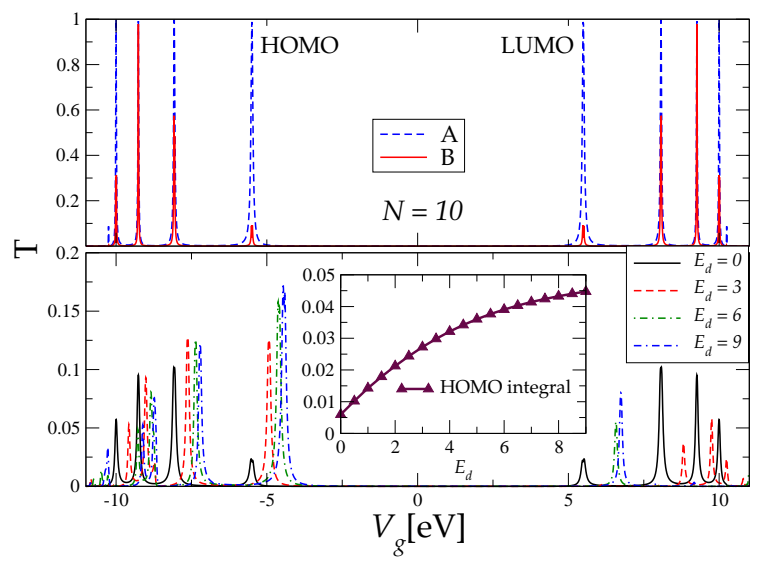

FIG. 3: Same as figure 2, for $N=10$ annulene.

ference can be totally destructive. For these annulenes the interference will be completely constructive in the A configuration and totally destructive if one lead is shifted by one site (B configuration, see Fig. 1). In these cases we can observe the emergence of a transmission channel, if the translational invariance is disrupted.

The effect of symmetry breaking by a local external perturbation is much more striking in annulenes with $4 n$ sites. As discussed above, we expect a new peak to emerge when the translational symmetry is broken. In figure 4 we show results for conductance through an 8site annulene represented by the PPP model. In the top figure, the HOMO and LUMO channels, fully formed for the A configuration, completely vanish in the $\mathrm{B}$ case due to quantum interference. When a local perturbation is

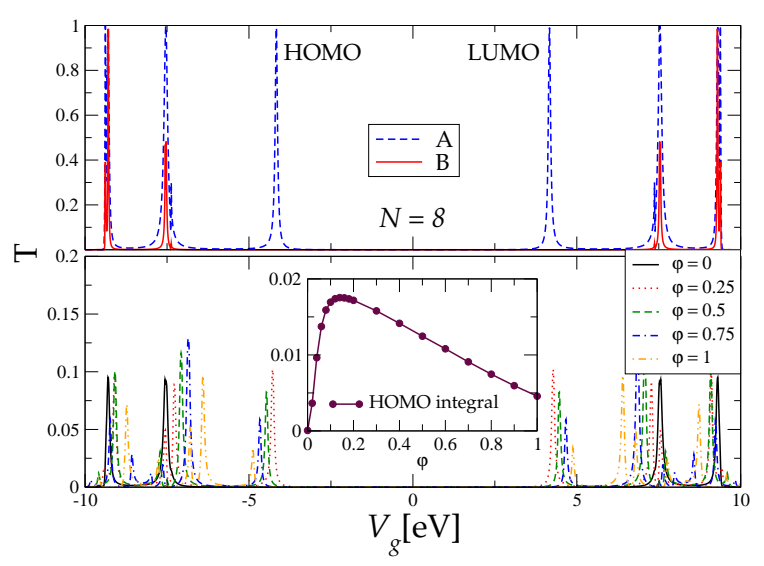

FIG. 4: Top: Transmittance through an [8]-annulene molecule for the A (broken line) and B (full line) configurations as a function of the gate voltage measured from the Fermi energy. Here $t^{\prime}=0.4$. Bottom: Transmittance for the B configuration in the presence of a local perturbation of the hopping of the form $t(1-\varphi)$ which breaks the translational invariance $(\eta=$ 0.03 and $\left.t^{\prime}=0.1\right)$. Inset: evolution of the HOMO integral as a function of $\varphi$. 

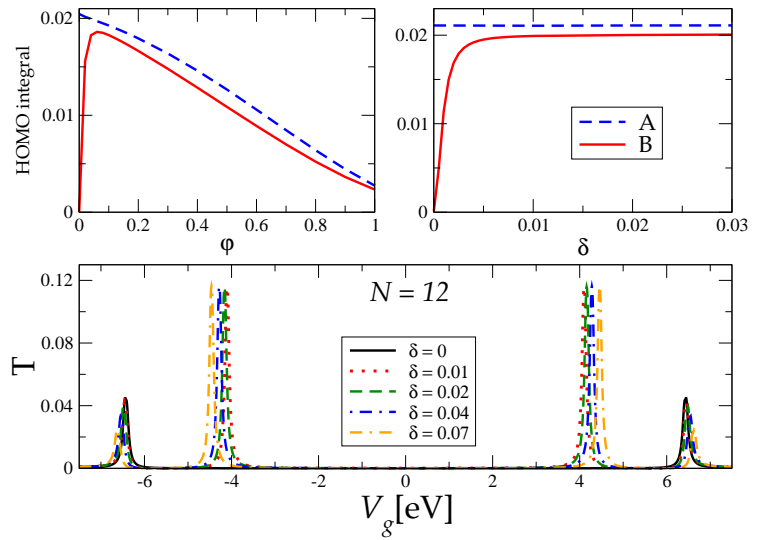

FIG. 5: Top: HOMO integral of a [12]-annulene molecule for the A (broken line) and B (full line) configurations as a function of the locally perturbed hopping $t(1-\varphi)$ (left panel) and the dimerization parameter $\delta$ (right panel). Bottom: Transmittance for the $\mathrm{B}$ configuration in the presence of dimerization.

applied to the molecule, in this case a different hopping on the bond to the right of the B lead, $t(1-\varphi)$, the absent peak emerges as shown in the inset of the bottom figure. A similar effect occurs also for a local potential

Finally, in figure 5 we present results for a [12]annulene. In this case also the HOMO and LUMO peaks for the B configuration are non-existent due to destructive interference. Here we show how the HOMO peak arises as a function of a different hopping parameter in one lead of the form $t(1-\varphi)$ (top left) and as a function of dimerization, $\delta$, for transfer integral for $t\left(1-(-1)^{i} \delta\right)$ of the bond between sites $i$ and $i+1$ (top right). The transmittance in the A configuration doesn't change in the presence of dimerization, while in case $\mathrm{B}$, the HOMO and LUMO channels develop suddenly already for a small $\delta$ (bottom panel). Dimerization of the annulenes lifts the two-fold degeneracy of the HOMOs as well as the LUMOs in large annulenes. Thus, the condition for destructive interference between the two paths is lifted and one observes transmission through these channels.

In conclusion, by analysing the resonant conductance through the HOMO and LUMO channels in the weak lead-molecule coupling regime we find a strong dependence on the source-drain configuration and on the molecular geometry due to quantum interference. This effect is more robust and striking than in the strongcoupling case since in the latter the destructive interference occurs only at zero gate voltage and can be masked in an experiment, while in the former a whole channel can appear or disappear depending on the geometry. We'd also like to stress that these results are not affected by molecular vibrations at room temperature since modes that can cause decoherence are excited at temperatures higher than $500 K[6]$.

In $[4 n+2]$-annulenes like benzene in the B ("meta") configuration (Fig. 1) the interference will be nearly destructive. The larger the molecule the closer the phase difference between different paths is to $\pi$ and hence the interference becomes more destructive. For [4n]annulenes, on the other hand, the interference for these configurations can be completely destructive. We have shown the effect of symmetry-breaking perturbations on different transmission channels. For $[4 n]$-annulenes the effect is striking since the transmission changes from zero to a large finite value for small perturbations. These effects should be seen in substituted annulenes and should also be appreciable in transport through rings of quantum dots.

We thank A. A. Aligia and B. Alascio for useful discussions. This work was done in the framework of projects PIP 5254 of CONICET, PICT 2006/483 of the ANPCyT and the ARG/RPO-041/2006 Indo-Argentine collaboration. SR thanks DST for support through JC Bose fellowship.

[1] A. Aviram, M. A. Ratner, Chem. Phys. Lett. 29, 277 (1974).

[2] M. A. Reed, et al., Science 278, 252 (1997).

[3] G. Cuniberti, G. Fagas, K. Richter (eds), Introducing Molecular Electronics, Springer, Berlin 2005.

[4] A. Nitzan, M. A. Ratner, Science 300, 1384 (2003).

[5] N. Tao, Nature Nanotech. 1, 173-181 (2006).

[6] D. Cardamone, et al., Nano Lett. 6, 2422 (2006).

[7] S-H. Ke, et al., Nano Lett. 8, 3257 (2008).

[8] S. Yeganeh, et al., Nano Lett. 9, 1770 (2009).

[9] J. Park, et al., Nature 417, 722-725 (2002).

[10] B. Venkataraman, et al., Nature 442, 904 (2006).

[11] A. V. Danilov, et al., Nano Lett. 8, 1 (2008).

[12] T. Dadosh, et al., Nature 436, 677 (2005).

[13] R. Pariser, R. Parr, J. Chem. Phys. 21, 466 (1953); J. A. Pople, Trans. of the Faraday Soc., 49, 1375 (1953).

[14] K. Ohno, Theor. Chim. Acta 2, 219 (1964).

[15] K. Hirose, "First-principles calculations in real space formalism: Electronic configurations and transport properties of nanostructures", Imperial College, London 2005.

[16] M. Büttiker, et al., Phys. Rev. B 31, 6207 (1985).

[17] For certain geometries (e.g. the meta configuration) the conductance at $V_{g}=0$ for the strong coupling regime can be zero due to quantum interference effects 25], but not between paths with $k=\pi / 2$ as stated in 6 , 7], since this is not an allowed quantum number for benzene molecules.

[18] T. Torelli, L. Mitas, Phys. Rev. Lett. 85, 1702 (2000).

[19] F. Sondheimer, Acc. Chem. Res. 5, 81 (1972).

[20] Z. G. Soos, and S. Ramasesha, Phys. Rev. B 29, 5410 (1984).

[21] E. Jagla, C. Balseiro, Phys. Rev. Lett. 70, 639 (1993).

[22] A. A. Aligia, et al., Phys. Rev. Lett. 93, 076801 (2004).

[23] S. N. Yaliraki and M. A. Ratner, Ann. N. Y. Acad. Sci. 960, 153 (2002).

[24] D. Walter, et al., Chem. Phys. 299, 139 (2004).

[25] G. Solomon, et al., Chem. Phys. 129, 054701 (2008).

[26] G. Begemann, et al., Phys. Rev. B 77, 201406(R) (2008); 78, 089901(E) (2008). 
[27] M. H. Hettler, et al., Phys. Rev. Lett. 90, 076805 (2003). 